\title{
Demanda física del portero de fútbol: necesidades y diferencias en respuesta al género
}

\section{Physical demand of the soccer goalkeeper: needs and differences in gender respons}

\author{
Boryi Alexánder Becerra-Patiño
}

`Universidad Pedagógica Nacional. Bogotá - Colombia. babecerrap@pedagogica.edu.co

Cómo citar: Becerra-Patiño, B.A. Demanda física del portero de fútbol: necesidades y diferencias en respuesta al género. Rev. Digit. Act. Fis. Deport. 7(1):e1526. http://doi.org/10.31910/rdafd.v7.n1.2021.1526

Artículo de acceso abierto publicado por Revista Digital: Actividad Física y Deporte, bajo una licencia Creative Commons CC BY-NC 4.0

Publicación oficial de la Universidad de Ciencias Aplicadas y Ambientales U.D.C.A, Institución de Educación Superior Acreditada de Alta Calidad por el Ministerio de Educación Nacional.

Recibido: Abril 23 de 2020 Aceptado: Octubre 29 de 2020 Editado por: Álvaro José Gracia Díaz

\section{RESUMEN}

Introducción: El estudio de la demanda física del portero de fútbol, obedece a unas necesidades y varía en respuesta con coherencia en las diferencias de género, por ello, su determinación en la competencia es necesaria. Objetivo General: Poder determinar el perfil de actividad y los requerimientos físicos (distancia total recorrida, aceleraciones, desaceleraciones, velocidad) en la competencia en los porteros/as de fútbol universitario en Bogotá a lo largo 64 partidos, implementando dispositivos GPS "FieldWiz" con una frecuencia de muestra de $(10 \mathrm{~Hz})$ para evaluar las diversas variables. Metodología: La población evaluada fueron 4 porteros de fútbol masculino y 4 porteras de fútbol femenina, y para ello, se evaluaron 8 partidos para cada portero y 8 partidos para cada portera. El tratamiento de los datos estadísticos se realizó mediante la implementación del programa $\mathrm{R}$, permitiendo la valoración y asociación de las variables, para que, en un primer momento en que se realizó un análisis descriptivo para las diversas categorías de locomoción se pudo conocer la distribución de la velocidad, así mismo, se realizó un análisis de varianza con dos indicadores (género y velocidad), a partir de la distancia recorrida, y que, fue calculada de forma euclediana entre los datos arrojados por el dispositivo GPS, y finalmente, se realizó un análisis de todas las variables (aceleración, desaceleración, distancia recorrida, sprint, velocidad, velocidad máxima y máxima aceleración) por medio de la prueba t para diferencia de medias para dos poblaciones (varones y mujeres, buscando encontrar las diferencias en función del género. Resultados: Los datos se presentan en DV, promedio y prueba $T$ para comprobar la validez de las muestras. Esta es una investigación con un enfoque cuantitativo, de carácter descriptivo y no experimental. Conclusiones: La investigación buscó abordar coherentemente, cuál es el perfil de actividad en el portero de fútbol universitario y las posibles diferencias en atención al género.

Palabras clave: Demanda física, Fútbol, Porteros, Evaluación de la competencia, Sistema gps.

\section{ABSTRACT}

Introducción: The study of the physical demand of the soccer goalkeeper, be due to some necessities and varies as a result of to gender differences, therefore its determination in competition is necessary. 
Objective: To be able to determine the activity profile and the physical requirements (total distance traveled, accelerations, decelerations, speed) in the competition in university soccer goalkeepers in Bogotá along 64 games, implementing GPS devices " FieldWiz "with a sample rate of $(10 \mathrm{~Hz})$ to evaluate the various variables. Methodology: The evaluated population were 4 male and 4 female soccer goalkeepers, for this 8 matches were evaluated for each goalkeeper and 8 matches for each goalkeeper. The treatment of the statistical data was made out through the implementation of the $\mathrm{R}$ program, allowing the valuation and association of the variables, for this, a descriptive analysis was initially carried out for the various categories of locomotion to know the speed distribution, and analysis of variance was also carried out with two indicators (gender and speed) based on the distance traveled and that It was calculated in a euclediana way among the data thrown by the GPS device and finally an analysis of all the variables (acceleration, deceleration, distance traveled, sprint, speed, maximum speed, and maximum acceleration) was carried out by means of the t-test for difference in average for two populations (men and women, seeking to find differences according to gender. Results: Lastly, the data are presented in DV, average $\mathrm{o}$ and T-test to check the validity of the samples. This is a quantitative investigation descriptive and non-experimental approachw. Conclusions: Hich seeks to coherently address the profile of activity in the university soccer goalkeeper and the possible differences in attention to gender.

Keywords: Physical demand,soccer, Goalkeeper, Competition assessment, gps system.

\section{INTRODUCCIÓN}

Elfútbol ha experimentado un crecimiento vertiginoso en todas sus dimensiones, principalmente, en aquellas estructuras relacionadas con el factor condicional, análisis de la velocidad, estudio exhaustivo de la distancia cubierta a diversas intensidades que ayuden a denotar el rol participativo de cada demarcación dentro de la competencia. En este sentido, el portero de fútbol cada vez cobra mayor protagonismo, en un juego de por sí, cada vez más dinámico, fluctuante e impredecible (Muñoz-Parreño, 2016; Gómez, 2011; Pol, 2011). El portero/a de fútbol es la posición dentro del terreno de juego, en el cual, sus necesidades dan respuesta a una serie de elementos técnicos, tácticos, reglamentarios, cognitivos, perceptivo-motrices y físicos, dado que su participación es fundamental, para garantizar el éxito de su equipo dentro de la competencia.

Hay que destacar que han sido múltiples los estudios que se han encargado de analizar el perfil físico del portero de fútbol dentro de la competencia, y que han logrado describir, de manera general, sus necesidades competitivas en respuesta a variables cinemáticas (Reilly \& Bowen, 1984; Bloomfield et al. 2006; Di Salvo et al. 2008; Condelo et al. 2011). Sin embargo, surge la necesidad no sólo conocer los requerimientos y condiciones, que debe poseer el portero de fútbol, sino que debemos ir más allá, y por eso, este estudio busca orientar y motivar la búsqueda de nuevas investigaciones centradas en el análisis de esta posición, y que respete, algunos principios generales del entrenamiento asociados con la participación activa y consciente, especificidad e individualidad. Otro aspecto a destacar son los sistemas de medición que se han empleado para categorizar los movimientos en función de la intensidad en la que el deportista se desplaza, estos estudios han utilizado sistemas de valoración manual (Reilly \& Bowen, 1984), sistema de análisis Prozone (Di Salvo et al. 2008) manteniendo un inconveniente a la hora de evaluar los movimientos, dado que es necesario, que la acción desarrollada por el deportista mantenga una velocidad promedio de (0,5 segundos), dificultad imperiosa de este sistema de valoración, debido a que, muchas de las acciones realizadas por el portero son cortas, explosivas y capaces de modificarse y transformarse en el tiempo (aceleración, velocidad media, velocidad máxima, desaceleración, cambios de ritmo, cambios de dirección, entre otros).

Otros instrumentos empleados para evaluar el rendimiento físico del portero de fútbol, es el video análisis software pro-motion, que emplea una multiplicidad de cámaras digitales de alta frecuencia y resolución, buscando computarizar la distancia que se recorre a diversas intensidades (Padulo et al. 2015). Finalmente, los sistemas de posicionamiento global GPS se han empleado para determinar el perfil de actividad, dispositivos que cuentan con una frecuencia de muestreo $(1 \mathrm{~Hz}, 10 \mathrm{~Hz}, 18 \mathrm{~Hz})$, y que permiten, monitorizar los rangos de velocidad de una manera eficaz, eficiente y efectiva (Castellano \& Casamichana, 2014), esta frecuencia de muestreo 
revela la calidad de los datos de referencia, principalmente, en aquellas acciones cortas y explosivas, y que demandan, cambios de dirección y velocidad (Witte $\&$ Wilson, 2004).

\section{MATERIALES Y METODOS}

La finalidad del presente estudio fue determinar las demandas de la competencia, y de esta manera, poder aportar al entrenamiento en especificidad del portero, y a su vez, brindar información útil sobre los requerimientos que demanda el juego para el portero según el género.

Variables. Las variables a evaluar son: distancia total recorrida, distancia cubierta en las diferentes zonas del terreno de juego según el seguimiento por mapeo de calor, distancia recorrida a diversas intensidades según la caracterización de las categorías de locomoción propuestas por Castagna et al. (2003) y Di Salvo et al. (2008), velocidad promedio y velocidad máxima, máxima aceleración, así como, cantidad total de aceleraciones y desaceleraciones en: 1,2 y $3 \mathrm{~m} / \mathrm{s}^{2}$ respectivamente.

Muestra. La muestra seleccionada comprende un (1) portero y una (1) portera de fútbol universitario de las cuatro instituciones más representativas de las ocho instituciones, que participan en el torneo masculino y 6 instituciones en el femenino organizado, por el SUE, y que cumplen, con los criterios de selección, entre las que se destacan obtención de los 4 primeros lugares en los distintos torneos universitarios, que se juegan en Bogotá, y contar, con un jugador/a en dicha posición que entrene como mínimo 3 veces a la semana, experiencia necesaria de al menos 2 años, jugando en esta posición, no haber sufrido alguna lesión, ni de miembros superiores, ni inferiores en los últimos 6 meses y no padecer de ninguna afectación de salud. El tamaño de la muestra es de 4 porteros y de 8 porteros en el torneo y 4 porteras de las 6 porteras, que compiten en este torneo.

Instrumentos de Medición y Técnicas. El instrumento empleado es un dispositivo GPS FieldWiz, utilizado para analizar y cuantificar el rendimiento deportivo de los deportistas de conjunto al aire libre, el cual es capaz de: 1.- Recibir, 2.- Almacenar y 3.- Procesar información para luego: Establecer y concretar las estadísticas recolectadas al final de la monitorización decada partido(Willmont, 2016). El uso de dispositivos
GPS "FieldWiz" con una frecuencia de muestreo GPS $10 \mathrm{~Hz}$ de alta precisión, acelerómetro de 9 ejes y 45gr., donde el deportista empleará un chaleco, con un sensor para determinar (zonas cubiertas en el campo, por seguimiento de mapa de calor, función de repetición de análisis de cualquier fase del juego, distancia recorrida, tiempo empleado en el campo, relación tiempo/distancia, estadísticas de velocidad, aceleraciones y carreras cortas "sprints". distancia, velocidad, aceleraciones, desaceleraciones, mapeo de calor).

El dispositivo se conecta a través de tecnología Bluetooth y se utiliza en la espalda. Este dispositivo GPS es de alta precisión, que brinda la posibilidad de transferir la información al final del juego o del entrenamiento, y cuenta con: Un software incluido (FieldWiz, Advanced Sport Instrument Sàrl, Avenue de Beaumont 51012 Lausana Suiza).

El proceso de recolección de los datos se desarrolló de la siguiente manera: 15 minutos antes del inicio de cada partido se colocó el chaleco con el dispositivo de posicionamiento satelital (GPS), que fue encendido para monitorizar el partido y fue retirado una vez concluido el mismo. La información fue transferida del GPS a la plataforma virtual, para lograr analizar las variables evaluadas, y finalmente, extraer los datos en un archivo Excel.

Procedimientos. En el presente estudio se utilizó el método investigativo de análisis de partidos a partir de la implementación de dispositivos GPS para analizar los partidos de fútbol universitario en Bogotá. Los indicadores analizados fueron: mapa de calor, distancia total recorrida, distancia cubierta en diferentes zonas, distancia recorrida a diversas intensidades según las categorías de locomoción propuestas por Castagna et al. (2003) y Di Salvo et al. (2008).

Todos estos datos son almacenados en la plataforma virtual de: FieldWiz, y luego, emitidos en un informe, para ser unificados en Excel, y finalmente, ser tratados. El tipo de procesamiento estadístico se realizó mediante el uso del programa $\mathrm{R}$ para programar análisis de carácter estadísticos y gráfico. El proceso de recolección de los datos se desarrolló de la siguiente manera: 15 minutos antes del inicio de cada partido, se colocó el chaleco con el dispositivo de posicionamiento satelital (GPS), 
que fue encendido para monitorizar el partido, y fue retirado, una vez concluido el mismo.

\section{RESULTADOS}

Los resultados del presente estudio se expresan de manera descriptiva, y para ello, se emplean una serie de gráficas y tablas que permitan dar respuesta a los objetivos planteados. Los datos fueron capturados mediante la utilización de instrumentos GPS (FieldWiz), es un sistema de seguimiento electrónico, y mide, el rendimiento físico y estratégico dedicado a deportes de conjunto como es en este caso, el fútbol.

Los resultados se presentan de manera descriptiva, por lo cual, se presenta una serie de gráficas y tablas que permitan dar respuesta a los objetivos planteados. Asimismo, la presentación de los resultados obtenidos está estructurada en los siguientes dos bloques:

1. Un primer momento destinado a encontrar las relaciones entre las categorías de locomoción. Este análisis inicial sirve para encontrar las relaciones que se establecen entre la distancia total recorrida, para ello, se hizo un análisis descriptivo para las diferentes categorías de velocidad definidas por Castagna et al. (2003) y Di Salvo et al. (2008), con el fin de conocer la distribución de la velocidad de los jugadores (porteros) durante los partidos, además se realizó un análisis de varianza con dos factores (género y categoría de velocidad) en función de la distancia recorrida, calculada de forma euclideana, entre las posiciones reportadas por: el GPS, lo cual, permitió determinar las diferencias por pares de categorías y entre género.

2. Se realizó un análisis de todas las variables (aceleración, desaceleración, distancia recorrida, velocidad, sprint, máxima aceleración y velocidad máxima), a través de la prueba t para diferencia de medidas para dos poblaciones (varones y mujeres), lo cual, permitía conocer los contrastes de cada una de las variables en función del género.

\section{Análisis de las categorías de locomoción de los porteros (as) de fútbol universitario en relación a su rol posicional. Uno de los principales objetivos del presente estudio era determinar la demanda física a partir de la distancia total recorrida y sus múltiples variables en dependencia de la intensidad, a la que se movilizan los porteros de fútbol, y encontrar, aquellas diferencias de acuerdo al género.}

En este sentido, las categorías de locomoción sirven para establecer unos rangos de velocidad, por lo cual, se puede visualizar sí, el deportista, en este caso, el portero de fútbol como posición específica, se moviliza en unos rangos de velocidad a lo largo de la competencia.

En las tablas 1 y 2, podemos establecer que las diferencias que subyacen de la distancia recorrida en diversos rangos de velocidad, en cada una de las categorías es significativa y que estas diferencias se agudizan, encontrándose una gran relevancia en los datos aportados, cuando se analizan en función del género, encontrando valores más elevados en los varones y en contrapartida a los expresados por las mujeres, y esto se encuentra relacionado con las capacidades condicionales y el rendimiento físico, que puede expresar la mujer deportista, dado que las respuestas fisiológicas, entre las que se destacan la frecuencia cardíaca y el consumo máximo de oxígeno, son asimiles en comparación con las evidenciadas en el fútbol masculino; esta versatilidad se agudiza más cuando se analizan los desplazamientos desarrollados a elevadas intensidades superiores a $15 \mathrm{~km} / \mathrm{h}$ (Barbero-Álvarez et al. 2009)

Tabla 1. Diferencias entre la distancia recorrida y distancia recorrida a diversas intensidades en función del género, según el análisis de las categorías de locomoción propuestas por Castagna et al. (2003).

\begin{tabular}{|c|c|c|c|c|c|c|}
\hline \multicolumn{7}{|c|}{ Categoría 1 } \\
\hline & Df & Sum Sq & Mean Sq & F value & $\operatorname{Pr}(>\mathrm{F})$ & \\
\hline Categoría 1 & 5 & 0,0106 & 0,0021164 & 17345,26 & $<2 \mathrm{e}-16$ & ${ }^{* * *}$ \\
\hline Sexo & 1 & 0 & 0,0000017 & 13,94 & 0,000189 & $* *$ \\
\hline Residuals & 2599950 & 0,3172 & 0,0000001 & & & \\
\hline
\end{tabular}

***Existen diferencias significativas entre la distancia recorrida en cada una de las categorías de velocidad

**Existen diferencias significativas entre la distancia recorrida por hombres y mujeres 
Tabla 2. Diferencias entre la distancia recorrida y distancia recorrida a diversas intensidades en función del género, según el análisis de las categorías de locomoción propuestas por Di Salvo et al. (2008).

\begin{tabular}{|c|c|c|c|c|c|c|}
\hline \multicolumn{7}{|c|}{ Categoría 2 } \\
\hline & Df & Sum Sq & Mean Sq & F value & $\operatorname{Pr}(>\mathbf{F})$ & \\
\hline Categoría 2 & 5 & 0,0088 & 0,0017668 & 14401,9 & $<2 \mathrm{e}-16$ & ${ }^{* \star \star}$ \\
\hline Sexo & 1 & 0 & 0,0000299 & 243,4 & $<2 \mathrm{e}-16$ & ${ }^{* \star}$ \\
\hline Residuals & 2599950 & 0,319 & 0,0000001 & & & \\
\hline
\end{tabular}

${ }^{* * *}$ Existen diferencias significativas entre la distancia recorrida en cada una de las categorías de velocidad

${ }^{* *}$ Existen diferencias significativas entre la distancia recorrida por hombres y mujeres

Todas las relaciones que se establecen a partir del análisis son significativas y basándonos en las categorías de locomoción propuestas por Di Salvo et al. (2008) y Castagna et al. (2003), aunque las categorías que mayor relevancia estadística revelan son las que denotan una diferencia $(-)$, todas las categorías analizadas muestran cierta significancia. Esto quiere decir, que el portero/a de fútbol recorre mayor distancia en la categoría parado y andando en relación con la carrera a intensidad máxima, y esto obedece, a la naturaleza de las acciones más recurrentes ejecutadas por el portero/a y su influencia dentro de la dinámica global del juego, su participación intermitente, así como, la mayor prevalencia de los equipos a mantener una defensa organizada y una organización defensiva para no recibir goles (De Baranda et al. 2005) (Tabla 3)

Tabla 3. Análisis de las categorías de locomoción según Castagna et al. 2003 y sus diferencias significativas entre categorías expresadas en $\mathrm{km}$.

\begin{tabular}{|l|c|c|c|c|}
\hline \multicolumn{1}{|c|}{ CATEGORÍA DE LOCOMOCIÓN 1 } & diff & Iwr & upr & p adj \\
\hline Andando-Parado & 0.0000641 & 0.0000626 & 0.0000656 & 0 \\
\hline Carrera baja intensidad-Parado & 0.0003518 & 0.0003438 & 0.0003597 & 0 \\
\hline Carrera intensidad media-Parado & 0.0005157 & 0.0004954 & 0.0005361 & 0 \\
\hline Carrera intensidad alta-Parado & 0.0002198 & 0.0002164 & 0.0002232 & 0 \\
\hline Carrera intensidad máxima-Parado * & -0.0000419 & -0.0000435 & -0.0000403 & 0 \\
\hline Carrera baja intensidad-Andando & 0.0002877 & 0.0002797 & 0.0002956 & 0 \\
\hline Carrera intensidad media-Andando & 0.0004516 & 0.0004313 & 0.0004720 & 0 \\
\hline Carrera intensidad alta-Andando & 0.0001557 & 0.0001523 & 0.0001591 & 0 \\
\hline Carrera intensidad máxima-Andando * & -0.0001060 & -0.0001076 & -0.0001043 & 0 \\
\hline Carrera intensidad media-Carrera baja intensidad & 0.0001639 & 0.0001421 & 0.0001857 & 0 \\
\hline Carrera intensidad alta-Carrera baja intensidad * & -0.0001320 & -0.0001405 & -0.0001235 & 0 \\
\hline Carrera intensidad máxima-Carrera baja intensidad * & -0.0003937 & -0.0004016 & -0.0003857 & 0 \\
\hline Carrera intensidad alta-Carrera intensidad media * & -0.0002959 & -0.0003165 & -0.0002753 & 0 \\
\hline Carrera intensidad máxima-Carrera intensidad media * & -0.0005576 & -0.0005780 & -0.0005372 & 0 \\
\hline Carrera intensidad máxima-Carrera intensidad alta * & -0.0002617 & -0.0002651 & -0.0002582 & 0 \\
\hline * Las diferencias más representativas son las que se encuentran en (-), aunque todas las diferencias son \\
\multicolumn{2}{|c|}{ significativas, pero estás diferencias son mínimas ya que se encuentran expresadas en km. } \\
\hline
\end{tabular}


En la carrera a baja intensidad, el portero cubre una mayor distancia en contraprestación con la que logra desarrollar en intensidad alta y máxima, lo que indica, que el nivel de la competencia, y la especificidad de esta demarcación dentro del terreno de juego, debe ser analizada en función de las acciones más recurrentes y las intensidades, a las cuales, se cubre esta distancia, debido a que, las acciones que realiza a máxima intensidad son acciones esporádicas mediadas por un juego co-adaptativo, y en el que también, debe estar preparado para realizar un gran abanico de posibilidades y tipos de desplazamientos.
Hay que destacar que las diferencias en esta posición de juego, siempre son más significativas, entre menor intensidad se ejecuten los desplazamientos, y esto responde, a que la distancia total recorrida a diversas intensidades, refleja que la naturaleza del portero de fútbol sea un jugador que permanece y cubra una mayor distancia en las categorías más bajas de locomoción (parado, andando), y que finalmente, las acciones a alta y máxima intensidad son las que determinan la eficacia de sus intervenciones; fortaleciendo la premisa referente a que, el fútbol es un deporte aeróbico por su duración, pero anaeróbico por la determinación de su resultado (Tablas 4 y 5).

Tabla 4. Diferencia de la distancia recorrida en función del género, según el análisis de las categorías de locomoción (Castagna et al. 2003).

\begin{tabular}{|c|c|c|c|c|}
\hline \multirow{2}{*}{ M-F } & diff & Iwr & upr & P adj \\
\cline { 2 - 5 } & 0,0000016 & 0,0000008 & 0,0000025 & 0,0002125 \\
\hline
\end{tabular}

Tabla 5. Diferencia de la distancia recorrida en función del género, según el análisis de las categorías de locomoción (Di Salvo et al. 2008).

\begin{tabular}{|c|c|c|c|c|}
\hline \multirow{2}{*}{ M-F } & diff & Iwr & upr & p adj \\
\cline { 2 - 5 } & 0,0000068 & 0,0000059 & 0,00000076 & 0 \\
\hline
\end{tabular}

Según los resultados obtenidos del análisis estadístico los varones recorren mayor distancia que las mujeres, y esto obedece, al nivel de la competencia, y específicamente, a las diferencias a nivel morfológico, fisiológico, y que finalmente, se configuran en las capacidades condicionales que permiten el movimiento del portero/a de fútbol.

Estas diferencias pueden explicarse debido a factores: 1.- Hormonales, 2.- Endócrinos, 3.- Antropométricos y 4.- Morfológicos (León, 2000), fisiológicos y metabólicos relacionados con un mayor porcentaje de hemoglobina y la eficiencia del gasto cardíaco (Shepard \& Astrand, 1996), condicionales relacionadas con la fuerza en términos absolutos (Baechle \& Earle, 2007), producción y generación de fuerza isocinética (Andrade et al. 2012; Hewett et al. 2008), el rendimiento aeróbico y respuesta respiratoria (Wilmore \& Costill, 2004) y las diferencias de la competencia condensada en la dinámica del juego, que es la que se encarga de proliferar un espectro amplio o reducido, de las acciones a desarrollar por el deportista (Andersson, 2014).

Las categorías de locomoción deben ser estudiadas a partir de rangos de velocidad específicos, para ello, se tomaron en consideración las propuestas por Castagna et al. (2003) debido al manejo, de niveles de intervención específicos a los desplazamientos realizados por: el portero y su intermitencia para intervenir dentro del juego y Di Salvo et al. (2008) al desarrollar una investigación sobre la distancia que recorren los porteros, específicamente, en la competencia. Esta formulación en ambos estudios produjo una escala de seis estructuras, que permiten establecer los rangos en los que se moviliza el deportista, estas categorías se presentan en la tabla 6. 
Tabla 6. Categorías de locomoción. Según Castagna et al. (2003) y Di Salvo et al. (2008).

\begin{tabular}{|c|c|c|}
\hline Categorías & $\begin{array}{c}\text { Valores Castagna et al. } \\
\mathbf{( 2 0 0 3 )}\end{array}$ & $\begin{array}{c}\text { Valores Di Salvo et al. } \\
\mathbf{( 2 0 0 8 )}\end{array}$ \\
\hline Categoría 1 & $0-0,4 \mathrm{~km} / \mathrm{h}$. & $0-0,2 \mathrm{~km} / \mathrm{h}$. \\
\hline Categoría 2 & $0,5-3 \mathrm{~km} / \mathrm{h}$. & $0,3-7,2 \mathrm{~km} / \mathrm{h}$. \\
\hline Categoría 3 & $3,1-8 \mathrm{~km} / \mathrm{h}$. & $7,3-14,4 \mathrm{~km} / \mathrm{h}$. \\
\hline Categoría 4 & $8,1-13 \mathrm{~km} / \mathrm{h}$. & $14,5-19,8 \mathrm{~km} / \mathrm{h}$. \\
\hline Categoría 5 & $13,1-18 \mathrm{~km} / \mathrm{h}$. & $19,9-25,2 \mathrm{~km} / \mathrm{h}$. \\
\hline Categoría 6 & $>18,1 \mathrm{~km} / \mathrm{h}$. & $>25,2 \mathrm{~km} / \mathrm{h}$. \\
\hline
\end{tabular}

Estudio de la distancia total recorrida y variables cinemáticas relacionadas con el rendimiento deportivo del portero de fútbol en respuesta al género. El análisis de la distancia total recorrida a diversas intensidades, y teniendo en consideración, las propuestas en dos estudios anteriores, hace que los rangos específicos para esta demarcación del terreno de juego, sea una necesidad imperante, y más aún, que estás deben estar orientadas en dependencia del género, dado que normalmente, lo que se establece es: medir y evaluar el fútbol femenino con los mismos parámetros del fútbol masculino (Andersson, 2010).
Las diferencias existentes entre la distancia total recorrida y analizando ambas investigaciones, estudio 1: (Castagna et al. 2003) y estudio 2: (Di Salvo et al. 2008), revela que las porteras recorren la mayor parte de su distancia en la categoría parado, en un rango de velocidad de $(0-0.4 \mathrm{~km} / \mathrm{h})$, que obedece a la naturaleza inquebrantable de su accionar, a la dinámica de lo impensado (Panzeri, 2011), a la poca participación de esta demarcación en la construcción del modelo de juego y su rol participativo en la competencia, y especialmente, a que el fútbol es de por sí, un juego espontáneo, mediado por las sinergias, emergencias y en el que predomina la incertidumbre (Tabla 7).

Tabla 7. Prevalencia de distancia recorrida por categorías de locomoción y prevalencia en función del género.

\begin{tabular}{|c|c|c|c|}
\hline Categoría 1 & Prevalencia & Categoría 2 & Prevalencia \\
\hline $\begin{array}{c}0-0,4 \mathrm{~km} / \mathrm{h} \\
\text { (Parado) }\end{array}$ & Mujeres & $\begin{array}{c}0-0,2 \mathrm{~km} / \mathrm{h} \\
\text { (Parado) }\end{array}$ & Hombres \\
\hline $\begin{array}{c}0,5-3 \mathrm{~km} / \mathrm{h} \\
\text { (Andando) }\end{array}$ & Hombres & $\begin{array}{c}0,3-7,2 \mathrm{~km} / \mathrm{h} \\
\text { (Andando) }\end{array}$ & Hombres \\
\hline $\begin{array}{c}3,1-8 \mathrm{~km} / \mathrm{h} \\
\text { (Carrera baja } \\
\text { intensidad) }\end{array}$ & Hombres & $\begin{array}{c}7,3-14,4 \mathrm{~km} / \mathrm{h} \\
\text { (Carrera baja } \\
\text { intensidad) }\end{array}$ & Hombres \\
\hline $\begin{array}{c}8,1-13 \mathrm{~km} / \mathrm{h} \\
\text { (Carrera } \\
\text { intensidad media) }\end{array}$ & Hombres & $\begin{array}{c}14,5-19,8 \mathrm{~km} / \mathrm{h} \\
\text { (Carrera } \\
\text { intensidad media) }\end{array}$ & Hombres \\
\hline $\begin{array}{c}13,1-18 \mathrm{~km} / \mathrm{h} \\
\text { (Carrera } \\
\text { intensidad alta) }\end{array}$ & Hombres & $\begin{array}{c}19,9-25,2 \\
(\text { Carrera } \\
\text { intensidad alta) }\end{array}$ & Hombres \\
\hline $\begin{array}{c}>18,1 \mathrm{~km} / \mathrm{h} \\
\text { (Carrera } \\
\text { intensidad máxima } \\
\text { o sprint) }\end{array}$ & Mujeres & $\begin{array}{c}>25,2 \mathrm{~km} / \mathrm{h} \\
\text { (Carrera } \\
\text { intensidad máxima } \\
\text { o sprint) }\end{array}$ & Mujeres \\
\hline
\end{tabular}


Por otra parte, la carrera de máxima intensidad o sprint es mayor cuando dicha categoría se contempla $>18.1 \mathrm{~km} / \mathrm{h}$ y se minimiza, cuando el valor de referencia es $>25.2 \mathrm{~km} / \mathrm{h}$, dilucidando que las acciones que ejecuta el portero de fútbol son cortas y explosivas, y, por ende, los recorridos que ejecuta no son superiores a los 10 metros, relación espaciotemporal que necesita para alcanzar su máxima velocidad y capacidad de aceleración.

El análisis de las acciones a baja intensidad está en correspondencia con el tipo de desplazamientos que demanda la competencia. En este sentido, la divergencia existente de la primera categoría locomotora de cada estudio hace que la propuesta de Castagna et al. (2003) las mujeres recorran una distancia mayor a la expresada por los varones en un rango que fluctúa entre los $(0-0.4 \mathrm{~km} / \mathrm{h})$, mientras que, la distancia que recorren los varones en la categoría de Di Salvo et al. (2008) es mayor en un rango de velocidad que comprende $(0-0.2 \mathrm{~km} / \mathrm{h})$ (Tabla 8).

Tabla 8. Estudio de las variables cinemáticas de distancia, velocidad, aceleración, desaceleración, sprint y máxima velocidad y aceleración en función de la prevalencia del género.

\begin{tabular}{|c|c|c|c|c|c|}
\hline Hombres-Mujeres & Diferencia promedio & p-valor & Significancia & Unidades & Ventaja \\
\hline Distancia total & 0.5076444 & $2.20 \mathrm{E}-06$ & si & $\mathrm{km}$ & Hombres \\
\hline Distancia $0-1 \mathrm{~km} / \mathrm{h}$ & -0.01060741 & 0.1180694 & no & $\mathrm{km}$ & \\
\hline Distancia 1-2km/h & 0.03348148 & 0.0839663 & no & $\mathrm{km}$ & \\
\hline Distancia 2-3km/h & 0.008696296 & 0.6071213 & no & $\mathrm{km}$ & \\
\hline Distancia 3-4km/h & 0.08316296 & 0.0061082 & si & $\mathrm{Km}$ & Hombres \\
\hline Distancia 4-5km/h & 0.1689926 & 0 & $\mathrm{si}$ & $\mathrm{Km}$ & Hombres \\
\hline Distancia 5-6km/h & 0.1028148 & 0 & $\mathrm{si}$ & $\mathrm{Km}$ & Hombres \\
\hline Distancia 6-7 km/h & 0.01438519 & 0.0110141 & $\mathrm{si}$ & $\mathrm{Km}$ & Hombres \\
\hline Distancia $7-8 \mathrm{~km} / \mathrm{h}$ & -0.000311111 & 0.9576628 & no & $\mathrm{Km}$ & \\
\hline Hid 12 km/h & 0.06342963 & $5.20 \mathrm{E}-06$ & $\mathrm{si}$ & $\mathrm{Km}$ & Hombres \\
\hline hid $15 \mathrm{~km} / \mathrm{h}$ & 0.02491111 & 4.47E-05 & si & $\mathrm{Km}$ & Hombres \\
\hline \# Acce $>1 \mathrm{~m} / \mathrm{s}^{\wedge} 2$ & 59.15407 & $7.64 \mathrm{E}-05$ & si & \# & Hombres \\
\hline \# Decel > 1 m / / $\mathrm{s}^{\wedge} 2$ & 97.42741 & 0.0111567 & si & \# & Hombres \\
\hline \# Acce $>2 \mathrm{~m} / \mathrm{s}^{\wedge} 2$ & 19.52074 & $2.00 \mathrm{E}-06$ & si & \# & Hombres \\
\hline \# Decel > $2 \mathrm{~m} / \mathrm{s}^{\wedge} 2$ & 16.97556 & $1.80 \mathrm{E}-06$ & si & \# & Hombres \\
\hline \# Acce $>3 \mathrm{~m} / \mathrm{s}^{\wedge} 2$ & 6.94963 & $3.00 \mathrm{E}-07$ & si & \# & Hombres \\
\hline \# Decel > $3 \mathrm{~m} / \mathrm{s}^{\wedge} 2$ & 2.700741 & 0.0110946 & si & \# & Hombres \\
\hline \# Sprints >13 km /h & 5.948889 & $8.90 \mathrm{E}-06$ & si & \# & Hombres \\
\hline \# Sprints >15 km /h & 3.721481 & $1.80 \mathrm{E}-06$ & si & \# & Hombres \\
\hline Avg Speed & 0.6219704 & $2.00 \mathrm{E}-07$ & si & $\mathrm{km} / \mathrm{h}$ & Hombres \\
\hline Max Speed & 2.754615 & 2.75E-05 & si & $\mathrm{km} / \mathrm{h}$ & Hombres \\
\hline Max Accel & 0.1556 & 0.4716606 & no & $\mathrm{m} / \mathrm{s}^{2}$ & \\
\hline
\end{tabular}

Cuando se estudia enfáticamente, que en la carrera a máxima intensidad, tanto para la categoría 1 (desplazamientos mayores a $18.1 \mathrm{~km} / \mathrm{h}$ ) y categoría 2 (acciones con una velocidad superior a $25.2 \mathrm{~km} / \mathrm{h}$ ), las porteras recorren una mayor distancia en este tipo de desplazamientos en comparación con los porteros, y esto, puede deberse a que en el fútbol masculino por una mayor competitividad y una organización defensiva más rigurosa, hace que el portero no realice tantas acciones, como si, se ejecutan en la práctica del fútbol femenino, debido a que, al existir un mayor espacio entre la línea defensiva y la portera, es mayor la cantidad de ataques con una duración menor y con una menor cantidad de pases en el fútbol femenino (Redondo et al. 2006), lo que puede influenciar lanzamientos a las espaldas de la línea defensiva y obligar a que la portera deba realizar desplazamientos largos, tanto en línea recta como en diagonal, fuera de su área alcanzando grandes velocidades, debido a que, en el fútbol femenino se recorre una mayor distancia precedida por pases largos y no por acciones individuales como: driblar, 
duelos individuales o pases cortos (Althoff et al. 2010), asimismo, cabe mencionar que al analizar el promedio de goles por partido, en los mundiales femeninos de: USA 1999, USA 2003 y China 2007 (3,5 gol por partido) versus el mundial masculino de Corea-Japón 2002 y Alemania 2006 (2,3 y 2,5 promedio de gol por partido respectivamente) la práctica del fútbol femenino refleja, una mayor participación de la portera en comparación con el rol que evidencia el portero (Castellano, 2008).

Si bien es cierto, que las diferencias en el género influyen de determinadas maneras, muchos son los procesos que deben considerarse a la hora de determinar el por qué, el deportista varón alcanza mejores rendimientos que la mujer en la mayoría de las variables analizadas en el presente estudio. Por otra parte, se observa que, en las categorías de locomoción de la distancia a intensidad variable, que comprende velocidades entre $(0-3$ y $7-8 \mathrm{~km} / \mathrm{h}$ respectivamente y máxima aceleración) no se encuentra significancia para inclinar la balanza a favor de varones o mujeres.

El compendio de otras acciones físicas, que recurrentemente el portero de fútbol debe ejecutar muestra como los varones consiguen valores superiores, en las acciones que comprenden las aceleraciones y desaceleraciones $\left(1,2\right.$ y $\left.3 \mathrm{~m} / \mathrm{s}^{2}\right)$ y sprint superiores a (13 y $15 \mathrm{~km} / \mathrm{h}$ ), así como de la velocidad media y máxima, reflejando una mayor capacidad contráctil por poseer una mayor masa muscular, debido a que, las mujeres al poseer niveles inferiores de testosterona, también ostentan un menor porcentaje de masa muscular total, y sí, es la masa muscular el elemento fundamental y determinante de la fuerza, el sexo femenino se encuentra en clara desventaja (Wilmore \& Costill, 2004), aunque, si bien es cierto, que está capacidad no obedece a factores coordinativos, encontrándose que en las acciones de carrera en distancias cortas, no existen grandes diferencias en respuesta al género (Weineck, 2005). Este es un interrogante que surge para entender el rendimiento del portero en atención al género, dado que es necesario comprender, tanto la totalidad como las interconexiones, que se establecen en todos los sistemas y subsistemas del ser humano.

\section{DISCUSIÓN}

El fútbol como deporte ha visto que el desarrollo tecnológico, se encuentra cada vez más vinculado a la misma práctica, tratando de encontrar aquellas respuestas relacionadas a la evaluación del rendimiento de los deportistas, esto ha permitido abonar un escenario para múltiples investigaciones, especialmente, aquellas relacionadas con determinar el perfil de actividad durante los partidos, a través de categorías como la distancia, velocidad, frecuencia cardíaca, entre otras.

Otro aspecto a destacar son los sistemas de medición, que se han empleado para categorizar los movimientos en función de la intensidad en la que el deportista se desplaza, estos estudios han utilizado sistemas de valoración manual (Reilly \& Bowen, 1984), sistema de análisis Prozone (Di Salvo et al. 2008) manteniendo un inconveniente, a la hora de evaluar los movimientos, dado que es necesario, que la acción desarrollada por el deportista mantenga una velocidad promedio de ( 0,5 segundos), dificultad imperiosa de este sistema de valoración, debido a que, muchas de las acciones realizadas por el portero son cortas, explosivas y capaces de modificarse y transformarse en el tiempo (aceleración, velocidad media, velocidad máxima, desaceleración, cambios de ritmo, cambios de dirección, entre otros). Otros instrumentos empleados para evaluar el rendimiento físico del portero de fútbol, es el video análisis software promotión, que emplea una multiplicidad de cámaras digitales de alta frecuencia y resolución, buscando computarizar la distancia que se recorre a diversas intensidades (Padulo et al. 2015).

Finalmente, los sistemas de posicionamiento global GPS se han empleado para determinar el perfil de actividad, dispositivos que cuentan con una frecuencia de muestreo $(1 \mathrm{~Hz}, 10 \mathrm{~Hz}, 18 \mathrm{~Hz})$ y que permiten monitorizar los rangos de velocidad de una manera eficaz, eficiente y efectiva (Castellano \& Casamichana, 2014), esta frecuencia de muestreo revela la calidad de los datos de referencia, principalmente, en aquellas acciones cortas $y$ explosivas y que demandan cambios de dirección y velocidad (Witte \& Wilson, 2004).

A diferencia de los jugadores de campo, en los porteros no se encuentra una disminución de la distancia cubierta a alta intensidad, tal como, si, se evidencia en los jugadores de campo (Mohr et al. 2003). Las acciones a alta intensidad que comprenden velocidades superiores a los $12 \mathrm{~km} / \mathrm{h}$ revelan valores de $(0.14 \pm 0.07$ y $0.14 \pm 0.08)$ y las acciones mayores a $15 \mathrm{~km} / \mathrm{h}(0.05 \pm 0.03$ y $0.06 \pm 0.03)$, 
para primer y segundo tiempo respectivamente, demostrando que este tipo de movimientos se mantiene, y esto puede obedecer, a la inconstante participación del portero y su perfil de actividad en el que predominan las acciones a baja intensidad (76.8\%), haciendo que, esté siempre en capacidad de participar activamente, y sin, una acumulación de fatiga importante, principalmente, en las acciones en las cuales tiene que intervenir, que al mismo tiempo son las situaciones que definen los partidos.

No se han encontrado estudios que evalúen y determinen la demanda física posicional para el portero de fútbol, descubriendo aquellas diferencias que pueden surgir en respuesta al género. Uno de los estudios que se encargó de evaluar esta premisa, halló que en el fútbol femenino élite se recorre una distancia similar a la de los varones (10-12km), donde la mayor diferenciación radica en la distancia total cubierta en rangos elevados de intensidad (Krustrup et al. 2005). Esto puede deberse a que tanto el rendimiento aeróbico como anaeróbico pueda verse afectado por el mayor tamaño de algunos órganos y masa corporal en el varón, y aunque, tanto varones como mujeres comprenden un promedio similar de fibras musculares oxidativas con preponderancia para trabajos de baja intensidad y glucolíticas para acciones de elevada intensidad, el tamaño de estas fibras musculares es mayor en los varones, facilitando un mayor aporte metabólico para la actividad deportiva (León, 2000).

La distancia total recorrida por parte de los porteros comprende distancias entre los 4.000 metros por partido (Reilly \& Bowen, 1984), mientras que otro estudio internacional que se encargó de evaluar 109 partidos determino que la distancia cubierta por cada juego era de 5,611 metros, de los cuales sólo el 2\% corresponden a acciones de alta intensidad superiores a $18 \mathrm{~km} / \mathrm{h}$ siguiendo las categorías de locomoción propuestas por Castagna et al. (2003). En el presente estudio los porteros cubrieron un promedio de distancia total por juego $(4.580 \pm 810 \mathrm{~m})$, donde todas las acciones realizadas por esta demarcación se encuentran asociadas a su rol participativo, así como a posibles alternativas y al continuum adaptativo del portero de fútbol en la competencia. En este sentido, al analizar las categorías de locomoción que comprenden las dimensiones de (parado, andando y carrera a baja intensidad) de $(0-8 \mathrm{~km} / \mathrm{h})$ se puede evidenciar que en este rango de intensidad se recorre $(3.52 \mathrm{~km} / \mathrm{h})$ representando el $76.8 \%$, mientras que en el estudio desarrollado por Di Salvo et al. (2003) la distancia recorrida a esta intensidad comprende el $73 \%$. El mayor porcentaje en acciones a baja intensidad encontradas en el presente estudio depende en gran medida a los factores contextuales, donde se desarrolla la práctica y al incremento de intervenciones que debe ejecutar el portero en función del nivel de la competencia.

La categorización de las acciones a alta intensidad depende de los rangos que se establecen para valorar los desplazamientos en función de la intensidad deseada, de esta misma forma, se encontró que el promedio total de acciones a alta intensidad fue 92, de las cuales 52 se realizaron hacia adelante y 40 hacia atrás (Padulo et al. 2015) y que están correlacionados con solo el $2 \%$ del compendio de movimientos totales en la competencia (Di Salvo et al. 2008). Estos datos revelan que en las acciones a alta intensidad que comprenden desplazamientos de velocidad superiores a los $13 \mathrm{~km} / \mathrm{h}$ y $15 \mathrm{~km} / \mathrm{h}$ correspondientemente se recorren $(0.30 \pm 0.14$ y $0.12 \pm 0.05 \mathrm{~km})$ en varones y $(0.16 \pm 0.10$ y $0.06 \pm 0.05)$ para mujeres y que la cantidad de sprints superiores a $13 \mathrm{~km} / \mathrm{h}$ y $15 \mathrm{~km} / \mathrm{h}$ fue de $(27.96 \pm 12.87$ y $16.04 \pm 6.72)$ y $(15.78 \pm 8.75$ y $7.78 \pm 5.96)$ para varones y mujeres, para un total de 44 acciones para el género masculino y 24 para el género femenino, demostrando que en las acciones a elevada intensidad la portera sólo ejecuta el $54 \%$ de las acciones que ejecuta el portero, factores que pueden obedecer al nivel de la competencia, promoción de ligas femeninas de alto nivel y con programas adaptados a las necesidades y adaptaciones del género femenino al continuum del juego (Andersson, 2010; Andersson, 2014).

Los porteros recorren en promedio $(4.58 \pm 0.81 \mathrm{~km})$ y (3.26 \pm 0.70$)$, de los cuales en un rango de velocidad de $(0-3 \mathrm{~km} / \mathrm{h})$ que comprende las dos primeras categorías de locomoción propuestas por Castagna et al. (2003) se recorren $(1.41 \mathrm{~km})$ y $(1.2 \mathrm{~km})$ representando el $30.7 \%$ y $36.7 \%$ de la distancia total recorrida para varones y mujeres respectivamente.

La cantidad de aceleraciones de $(1 \mathrm{~m} / \mathrm{s}, 2 \mathrm{~m} / \mathrm{s}$ y $3 \mathrm{~m} / \mathrm{s}^{2}$ ) son las acciones que más se proliferan alcanzando valores de: $(446 \pm 93 ; 109-74 \pm 28.77$; $28.65 \pm 11.41)$ y $(288.8 \pm 100 ; 61.04 \pm 26.88 ; 11.67 \pm 8)$ por juego, mientras que la cantidad de acciones de desaceleración de $\left(1 \mathrm{~m} / \mathrm{s}, 2 . \mathrm{m} / \mathrm{s}\right.$ y $\left.3 \mathrm{~m} / \mathrm{s}^{2}\right)$ fueron $(413 \pm 94 ; 86.83 \pm 26.61 ; 23.78 \pm 5.74)$ y $(251.9 \pm 101$; $49.8 \pm 23$; $14.7 \pm 9.5)$ para varones y mujeres 
correspondientemente, este tipo de acciones, son las que le permiten al portero realizar la mayor parte de sus intervenciones, asociado también, a la manera de posicionar la mano a la altura de la rodilla, contribuyendo a poder desviar los remates que provienen del lado siniestro y diestro del área de penalización, dado que esta posición favorece la efectividad, eficacia y eficiencia, sí, los remates son lanzados desde áreas proximales. La velocidad promedio del portero se encuentra en un rango que oscila entre $(3.01 \pm 0.46 \mathrm{~km} / \mathrm{h})$ para varones y para mujeres (2.31 \pm 0.51$)$, la máxima velocidad alcanzada en promedio fue $(23.47 \mathrm{~km} / \mathrm{h})$ y $(19.71 \pm 2.89)$ para varones y mujeres respectivamente y la máxima aceleración en $\left(\mathrm{m} / \mathrm{s}^{2}\right)$ fue $(4.74 \pm 0.48)$ para el portero masculino, mientras que, la portera femenina alcanzó valores de: (4.08 \pm 0.53$)$. Estas diferencias reflejan que las necesidades imperantes de la competencia proveen no sólo diferencias interindividuales, sino intraindividuales que deben considerarse a la hora de evaluar el rendimiento de los patrones de actividad locomotriz, para este puesto específico en el terreno de juego.

La distancia en promedio en el primer tiempo fue de $(2.39 \pm 0.44)$ y $(1.74 \pm 0.37)$, representando el $52 \%$ y (53\%) para varones y mujeres correspondientemente. La distancia recorrida, y que comprende, las acciones a baja intensidad (parado, andando y carrera a baja intensidad) revela que se recorre más distancia en el primer tiempo en contrapartida a lo expresado en el segundo tiempo sin discriminación del género.

Las acciones de aceleración que comprenden unas velocidades de ( 1 y $2 \mathrm{~m} / \mathrm{s}^{2}$ ) son superiores en el primer tiempo (244.5 \pm 49.24 y $56.35 \pm 15.6)$ para varones, y que disminuyen, en el segundo tiempo (204.9 \pm 56.8 ; $190.1 \pm 51.8)$, las acciones de aceleración de $3 \mathrm{~m} / \mathrm{s}^{2}$ son mayores en el segundo tiempo (15.04 \pm 7.2 vs $13.5 \pm 5.9)$ en varones, mientras que, en mujeres las acciones de aceleración de $\left(1,2\right.$ y $\left.3 \mathrm{~m} / \mathrm{s}^{2}\right)$ son mayores en el primer tiempo $(159.1 \pm 62.5 ; 35.15 \pm$ $14.8 ; 6.26 \pm 5)$, y que disminuyen, en el segundo tiempo $(129.5 \pm 53.4 ; 25.8 \pm 15.3 ; 5.41 \pm 4.3)$.

Por otro lado, las acciones de desaceleración revelan que en situaciones de ( 1 y $\left.2 \mathrm{~m} / \mathrm{s}^{2}\right)$ los valores obtenidos en el primer tiempo son superiores, mientras que, los valores de $\left(3 \mathrm{~m} / \mathrm{s}^{2}\right)$ son mayores en el segundo tiempo para varones y en las mujeres las acciones de desaceleración de $(1,2$ y $3 \mathrm{~m} / \mathrm{s} 2)$ son mayores en el primer tiempo, y que disminuyen, a medida que el partido llega a su tramo final. Esto evidencia: 1.- La intermitencia, 2.- Imprevisibilidad e 3.- Incertidumbre con la que el deportista debe aprender a convivir en esta posición dentro del campo de juego.

\section{CONCLUSIONES}

\section{El presente estudio encontró las siguientes conclusiones:}

Los perfiles de actividad de los porteros deben considerar unas categorías de locomoción que establezcan rangos de velocidad específicos a las necesidades de su posicionamiento.

Las diferencias de género revelan que el portero masculino desarrolla una mayor distancia total recorrida, tanto en primer como en segundo tiempo, mayor distancia desarrollada a elevada intensidad $(>15 \mathrm{~km} / \mathrm{h})$, mayor cantidad de aceleraciones y desaceleraciones en $\left(1,2\right.$ y $\left.3 \mathrm{~m} / \mathrm{s}^{2}\right)$, así como, una máxima velocidad y máxima aceleración que la portera femenina.

La demarcación del portero de fútbol universitario en Bogotá en partidos de (90 minutos) recorre una distancia de $(4,58 \mathrm{~km})$ para varones y $(3,26 \mathrm{~km})$ para mujeres, por lo que, la portera sólo desarrolla un $71 \%$ de la distancia realizada por el portero.

En el primer tiempo la portera de fútbol alcanza mejores resultados en distancia recorrida a diversas intensidades $(0-8 \mathrm{~km} / \mathrm{h})$, cantidad de sprints superiores a 13 y $15 \mathrm{~km} / \mathrm{h}$, acciones de aceleración, desaceleración, máxima velocidad y máxima aceleración, mientras que, el portero de fútbol alcanza mejores resultados en sprints superiores a $13 \mathrm{~km} / \mathrm{h}$., aceleración de $3 \mathrm{~m} / \mathrm{s}^{2}$, desaceleración de $3 \mathrm{~m} / \mathrm{s}^{2}$, y máxima velocidad en el segundo tiempo. La capacidad para desarrollar este tipo de acciones está relacionada con la experiencia del/la deportista, el rol táctico, el género y el nivel de la competencia.

El fútbol como deporte intermitente, cargado de incertidumbre y constantes fluctuaciones demuestra que las necesidades del juego deben ir en correlación con los principios de especificidad e individualidad en esta posición, y en respuesta a las diferencias de género, debido a que, la mujer 
realiza el $71 \%$ de la distancia total recorrida, el $53.3 \%$ de las acciones superiores a $12 \mathrm{~km} / \mathrm{h}, 50 \%$ de los desplazamientos superiores a $15 \mathrm{~km} / \mathrm{h}$, el 64, 56 y $39 \%$ de las acciones de aceleración de 1,2 y $3 \mathrm{~m} / \mathrm{s}^{2}$ respectivamente, el 61,57 y $61 \%$ de las desaceleraciones en 1,2 y $3 \mathrm{~m} / \mathrm{s}^{2}$, el $76.7 \%$ de la velocidad promedio, el $83.9 \%$ de la máxima velocidad y el $86 \%$ de la máxima aceleración que el hombre desarrolla. Esto nos da a entender que el fútbol femenino necesita ser entendido, bajo una lógica que respete sus propias capacidades y necesidades competitivas.

Este es el primer estudio dirigido a: analizar, evaluar y determinar el perfil de actividad desde una óptica físico-táctica en los porteros de fútbol en Bogotá, premisa por la cual, esta investigación pretende servir como punto de inflexión para poder generar más estudios.

Limitaciones. La principal limitación de la investigación está relacionada con las interrelaciones que hicieron falta por establecerse entre los desplazamientos a diversas intensidades reflejados en la distancia total recorrida como acción física, y las coordenadas polares, que revelan aquellas zonas de influencia y confluencia en las cuales participó el portero en la competencia como acción táctica. Sin embargo, la no contemplación de la eficacia de las intervenciones realizadas a partir de la interacción de las estructuras coordinativa, cognitiva y socioafectiva, no permiten comprender la complejidad y continua co-adaptatividad que debe proliferarse en el portero de fútbol. Asimismo, otra problemática surge al no haber analizado algún indicador fisiológico (frecuencia cardiaca, lactato, entre otros), para así, lograr una caracterización holística del perfil de actividad del portero en función de las necesidades y similitudes de género. Todos estos elementos, deben ser incorporados poco a poco para futuras investigaciones.

Recomendaciones. La siguiente distribución de la distancia cubierta a diversas intensidades revela que las acciones de mínima intensidad y poca velocidad son mayores en mujeres, mientras que, a medida que la intensidad se incrementa a partir de los $(3.1 \mathrm{~km} / \mathrm{h})$ el hombre registra mejores rendimientos. Esta especificidad de una misma posición en respuesta a las diferencias y necesidades del género, abre una brecha importante para proponer unas categorías de locomoción adaptadas a los requerimientos de la: 1.-Distancia, 2.- Velocidad y 3.- Intensidad con la que se desplaza el portero/a de fútbol (Figura 1).

Figura 1. Propuesta para las categorías de locomoción en función del género y análisis de la distancia total recorrida a diversas intensidades. Adaptado de Castagna et al. (2003) y Di Salvo et al. (2008).

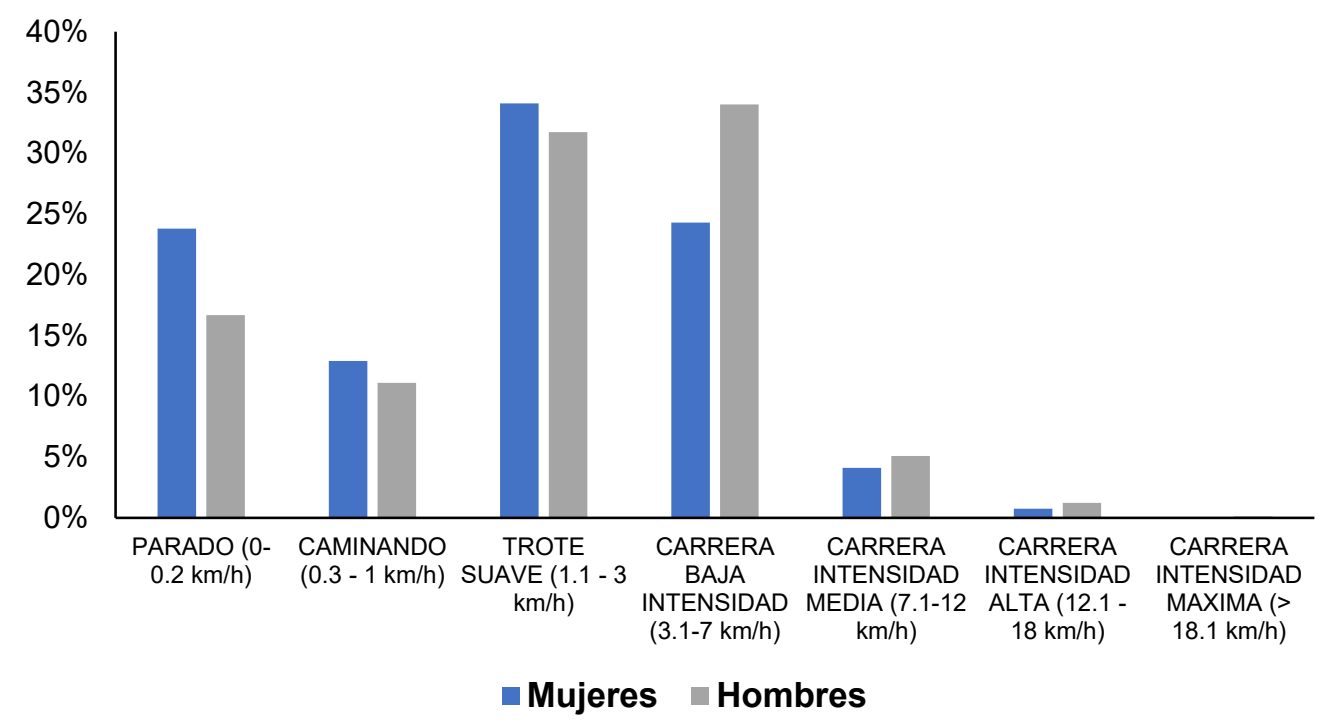


Uno de los puntos más importantes en la consideración del análisis del perfil de actividad del portero de fútbol, es que las categorías de velocidad deben ir asociadas a la intensidad, con la que realiza la diversidad de sus movimientos. En este sentido, el presente estudio intentó describir la demanda física del portero de fútbol universitario en Bogotá, en atención a las necesidades y diferencias del género, y con base en ello, poder adaptar unas categorías de locomoción que respondan a las particularidades del contexto, al nivel de la competencia y a los requerimientos que establece el juego, y que, condiciona a cada jugador.

Las categorías de locomoción específica teniendo en consideración el análisis de sus desplazamientos se sustenta en siete categorías descritas así:

-Categoría 1: "Parado" (0-0,2km/h).

-Categoría 2: "Caminando" (0,3-1km/h).

-Categoría 3: "Trote" (1,1-3km/h).

-Categoría 4: "Carrera a baja intensidad" (3,1-7km/h). -Categoría 5: "Carrera intensidad media" (7,1-12km/h). -Categoría 6: "Carrera intensidad alta" (12,1-18km/h). -Categoría 7: "Carrera intensidad máxima" (> 18,1 km/h).

La sugerencia de estas categorías surge de la necesidad por comprender la naturalidad del portero de fútbol, en atención a su propia especificidad e individualidad, y a partir de allí, encontrar la manera en: cómo los datos que se sustraen son relevantes para buscar interrelacionar la infinita gama de desplazamientos, que puede ejecutar el portero dentro de su rol participativo.

El aporte de la presente investigación representa un primer paso para poder comprender, que las demandas competitivas en función del género en el portero/a del fútbol son un primer paso a la hora de: 1.- Organizar, 2.- Direccionar, 3.- Programar y 4.Ejecutar, el proceso de entrenamiento. Asimismo, está es una primera aproximación dirigida a describir y determinar los requisitos que provee el juego en respuesta al género en esta demarcación, abriendo la posibilidad al desarrollo de nuevos estudios que contemplen que las categorías de locomoción, deben ajustarse a las posibilidades de intervención de esta posición en la competencia.

\section{REFERENCIAS}

1. ALTHOFF, K.; KROIHER, J.; HENNING, E. 2010. A soccer game analysis of two World Cups: playing behavior between elite female and male soccer players. Footwear Science. 2(1):51-56.

https://doi.org/10.1080/19424281003685686

2. ANDERSSON, H. 2010. The physiological impact of soccer on elite female players and the effects of active recovery training. Örebro universitet (Örebro). 70p.

3. ANDERSSON, H. 2014. Training for performance: Developing elite female football players. SISU Idrottsböcker och författarna. (8):1-11.

4. ANDRADE, M.; DE LIRA, C.; KOFFES, F.; MASCARÍN, N.; BENEDITO-SILVA, A.; DA SILVA, A. 2012. Isokinetic hamstrings-to-quadriceps peak torque ratio: the influence of sport modality, gender, and angular velocity. J Sports Sci. 30(6):547-553.

https://doi.org/10.1080/02640414.2011.644249

5. BAECHLE, T.; EARLE, R. 2007. Principios del entrenamiento de la fuerza y del acondicionamiento físico. Médica Panamericana (Madrid, España).

6. BARBERO-ÁLVAREZ, J.; COUTTS, A.; GRANDA, J.; BARBERO, V.; CASTAGNA, C. 2009. The validity and reliability of a global positioning satellite system device to assess speed and repeated sprint ability (RSA) in athletes. Journal of Science and Medicine in Sport. 13(2):232-235. https://doi.org/10.1016/j.jsams.2009.02.005

7. BLOOMFIELD, J.; POLMAN, R.; O'DONOGHUE, P. 2006. Physical demands of different positions in FA Premier League soccer. Journal of Sports and Medicine. 6(1):63-70.

8. CASTAGNA, C.; D'OTTAVIO, S.; ABT, G. 2003. Activity profile of young soccer players during actual match play. Journal of Strength and Conditioning Research. 17(4):775-780.

9. CASTELLANO, J. 2008. Fútbol e Innovación. Wanceulen (Sevilla, España). 223p.

10. CASTELLANO, J.; CASAMICHANA, D. 2014. Deporte con dispositivos de posicionamiento global (GPS): Aplicaciones y limitaciones. Revista de Psicología del Deporte. 23(2):355-364. 
11. CONDELO, G.; LUPO, C.; CIPRIANI, A.; TESSITORE, A. 2011. Activity profile of a no-professional goalkeeper during oficial matches. Annals of Research in sport and physical activity. 2:94-95.

12. DE BARANDA, P.; ORTEGA, E.; LLOPIS, L.; NOVO, J.; RODRÍGUEZ, D. 2005. Análisis de las acciones defensivas del portero de fútbol 7. Apunts de Educación Física y Deportes. 80:45-52.

13. DI SALVO, V.; BENITO, P.; CALDERÓN, M.; DI SALVO, M.; PIGOZZI, F. 2008. Activity profile of elite goalkeepers during football match-play. J Sports Med Phys Fitness. 48(4):443-446.

14. GÓMEZ, P. 2011. La preparación física del fútbol contextualizada en el fútbol. McSports (Pontevedra). 312p.

15. HEWETT, T.; MYER, G.; ZAZULAK, B. 2008. Hamstrings to quadriceps peak torque ratios diverge between sexes with increasing isokinetic angular velocity. J Sci. Med Sport. 11(5):452-459.

https://doi.org/10.1016/j.jsams.2007.04.009

16. KRUSTRUP, P.; MOHR, M.; ELLINGSGAARD, H.; BANGSBO, J. 2005. Physical demands during an elite female soccer game: importance of training status. Medicine and science in sports and exercise. 37(7):1242-1248.

https://doi.org/10.1249/01.mss.0000170 062.73981 .94

17. LEÓN, C. 2000. Influencia del sexo en la práctica deportiva. Biología de la mujer deportista. Arbor. 165(650):249-263. https://doi.org/10.3989/arbor.2000.i650.968

18. MOHR, M.; KRUSTRUP, P.; BANGSBO, J. 2003. Match performance of high-standard soccer players with special reference to development of fatigue. Journal of sports sciences. 21(7):519-528.

https://doi.org/10.1080/0264041031000071182

19. MUÑOZ-PARREÑO, J. 2016. Planificación estratégica de la temporada, Tomo 1: Paradigmas, modelo de juego y metodología. McSports (Vigo, España). 192p.

20. PADULO, J.; HADDAD, M.; ARDIGÓ, L.; CHAMARI, K.; PIZZOLATO, F. 2015. High frequency performance analysis of professional soccer goalkeepers: A pilot study. J Sports Med Phys Fitness. 55(6):557-562.
21. PANZERI, D. 2011. Fútbol. Dinámica de lo impensado. Capital Swing Libros (Madrid, España). 320p.

22. POL, R. 2011. La preparación ¿física? en el fútbol: el proceso de entrenamiento desde las ciencias de la complejidad. McSports (Pontevedra, España). 210p.

23. REILLY, T.; BOWEN, T. 1984. Exertional cost of changes in directional modes of Running. Perc Motor Skills. 55:267-271.

https://doi.org/10.2466\%2Fpms.1984.58.1.149

24. REDONDO, J.; CUADRADO, G.; DE BENITO, A.; SEDADO, S. 2006. Análisis comparativo de la acción de juego en el último tercio de cada parte en un partido de fútbol (fútbol femenino vs. fútbol masculino). RED. 20(2):33-42.

25. SHEPARD, R.; ASTRAND, P. 1996. La resistencia en el deporte. Paidotribo (Barcelona, España). $668 p$.

26. WEINECK, J. 2005. Entrenamiento total. Paidotribo (Barcelona, España). 688p.

27. WILLMONT, A. 2016. FieldWiz GPS pilot Research report. Sport and Exercise Science Consultancy Unit. 23p.

28. WILMORE, J.; COSTILL, D. 2004. Fisiología del esfuerzo y del deporte. Paidotribo (Barcelona, España). 776p.

29. WITTE, T.; WILSON, A. 2004. Accuracy of nondifferential GPS for the determination of speed over ground. Journal of biomechanics. 37(12):1891-1898.

https://doi.org/10.1016/j.jbiomech.2004.02.031 